\title{
Power Control for Satellite Uplink and Terrestrial Fixed-Service Co-existence in Ka-band
}

\author{
Eva Lagunas, Shree Krishna Sharma, Sina Maleki, Symeon Chatzinotas, Björn Ottersten \\ Interdisciplinary Centre for Security, Reliability and Trust (SnT) \\ University of Luxembourg, 4 rue Alphonse Weicker, L-2721, Luxembourg \\ Email: \{eva.lagunas,shree.sharma,sina.maleki,symeon.chatzinotas,bjorn.ottersten\}@uni.lu
}

\begin{abstract}
A fundamental problem facing the next generation of Satellite Communications (SatComs) is the spectrum congestion and how the scarce spectral resources are allocated to meet the demand for higher rate and reliable broadband communications. In this context, this paper addresses the satellite uplink where satellite terminals reuse frequency bands of FixedService (FS) terrestrial microwave links which are the incumbent users in the Ka band. In this scenario, the transmit power of the satellite terminals has to be controlled such that the aggregated interference caused at the FS system is kept below some acceptable threshold. In this paper, we review simple and efficient power allocation techniques available in the literature and, with slight adaptations, we evaluate them to the proposed satellite uplink and terrestrial FS co-existence scenario. The presented numerical results highlight the tradeoff between the level of channel state information and the rates that can be achieved at the satellite network.
\end{abstract}

\section{INTRODUCTION}

The Digital Agenda for Europe (DAE) [1] sets forth ambitious requirements for broadband communications, in which Satellite Communications (SatCom) play a key role [2]. The relatively new High Throughput Satellites (HTS) operating in Ka-band, like Eutelsats KaSat, VIASAT 1 and SES-12, have been shown to provide system capacity up to $100 \mathrm{Gbps}$, which is still far from the challenging objectives set forth by the European Commission. In particular, it is foreseen that each user will demand 30-40 Mbps and even up to $100 \mathrm{Mbps}$ by 2020, mainly as a result of the expected Internet and video demand growth [3]. The main limiting factor in providing these user rates is the exclusive spectrum assigned to Fixed Satellite Service (FSS), which is of $500 \mathrm{MHz}$ in both the up and downlinks.

The application of Cognitive Radio (CR) techniques [4] in SatComs has recently received significant attention in both academia and industry as an innovative approach that improves the spectrum utilization and alleviates the spectrum scarcity [5]-[8]. In CR networks, there are two types of systems. One is the incumbent system and the other is the cognitive system which is allowed to operate on the currently allocated spectrum as long as the incumbent communications are not adversely affected. In this paper, we address the cognitive satellite uplink where satellite terminals reuse frequency bands of FixedService (FS) terrestrial microwave links as the incumbent users in the $\mathrm{Ka}$ band $(27.5-29.5 \mathrm{GHz})$, which corresponds to one of the preselected scenarios in the FP7 CoRaSat project [6].

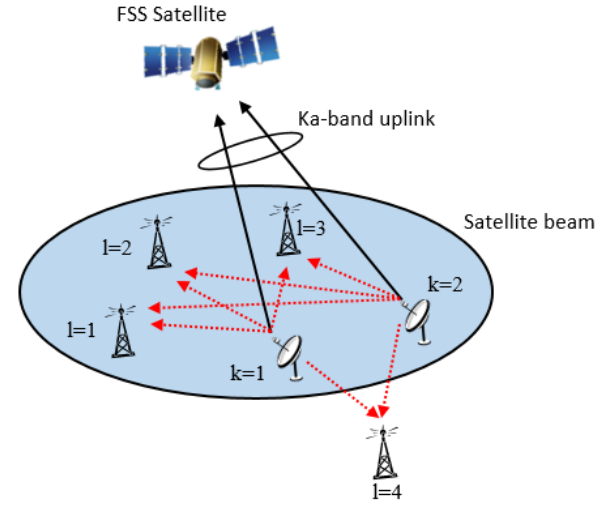

Fig. 1: Spectral coexistence of FSS uplink with FS terrestrial system in the Ka-band

Fig. 1 depicts the considered scenario, in which the transmission power constitutes an important resource that should be properly controlled in order to protect the FS systems from harmful interference due to the FSS terminals uplink transmission. This paper focuses on a single satellite scenario but the presented techniques can be easily extended to multiple satellite scenarios.

In the underlay CR systems, which is the focus of this paper, outage probability is usually used to assess the quality of the incumbent communications [9], [10]. However, the outage metric does not instantaneously protect the incumbent system because it has to be averaged over a period of time [11]. The alternative approach is to use an interference constraint limit that the licensed receiver can get from the cognitive system [12]. This interference limit naturally determines the maximum power which the cognitive FSS terminal can transmit. The goal of this paper is to optimally allocate the transmit power of the cognitive FSS terminal devices ensuring that its transmissions do not adversely interfere with the incumbent FS system. Power control for interference management has been successfully applied in general interference channels like the underlay CR scenario [13] and the Time-Area-Spectrum (TAS) licensed [14], [15]. Efficient power allocation algorithms for the cognitive uplink satellite-terrestrial co-existence, in contrast, have not received much attention in the literature. It should be noted that cognitive satellite uplink is different from a conventional interference channel since the interference from 
the FS terrestrial system to the satellite can be neglected due to large distance between them as well as the directivity of the Ka band terrestrial communications [16]. The applicability of $\mathrm{CR}$ in the aforementioned scenario was discussed in [17], concluding that both satellite and terrestrial systems could potentially operate in the same band without degrading each others performance. Here, we review simple and efficient power allocation techniques available in the literature and adapt them to the proposed satellite uplink and terrestrial FS co-existance scenario. In particular, we state and compare through simulation experiments three different transmit power allocation strategies, depending on the amount of side information available at each satellite terminal.

The remainder of this paper is organized as follows. Section II introduces the signal model and the problem statement. Section III investigates the achievable power and rates tuples. Then, three power control schemes are adapted and discussed in Section IV. Section V presents the numerical results, and Section VI concludes the paper.

\section{Signal Model and Problem Statement}

We consider a scenario with $K$ FSS terminal users and $L$ FS microwave links. The transmit power of the $k$-th FSS terminal is $p_{k}$ and we define $\mathbf{p}=\left[\begin{array}{llll}p_{1} & p_{2} & \ldots & p_{K}\end{array}\right]^{\mathrm{T}}$ as the power allocation vector. Moreover, the maximum transmit power for the $k$-th FSS terminal is denoted by $P_{k}^{\max }$ and we define $\mathbf{P}^{\max }=\left[\begin{array}{llll}P_{1}^{\max } & P_{2}^{\max } & \ldots & P_{K}^{\max }\end{array}\right]^{\mathrm{T}}$. Let the channel power gains of the interference links be $g_{k, l}=\left|h_{k, l}\right|^{2}$, with $h_{k, l}$ being the instantaneous cross-channel coefficient from the $k$-th FSS terminal to the $l$-th FS station. The interference links of a simplified satellite-terrestrial co-existence network with $K=2$ FSS terminals and $L=3$ FS stations are shown in Fig. 2.

Assuming single user detection, the achievable rate by the $k$-th FSS terminal is given by,

$$
C_{k}=\log _{2}\left(1+\frac{d_{k} p_{k}}{\sigma^{2}}\right),
$$

where $d_{k}=\left|h_{k}\right|^{2}$ denotes the instantaneous channel power gain of the link from the $k$-th FSS terminal to the satellite. This includes transmit and receive antenna gains and propagation loss. In (1), it has been assumed that the noise power level, $\sigma^{2}$, is equal for all receivers. We consider through the paper $\sigma^{2}=1$.

One of the major challenges for cognitive uplink satellite communications is to balance between maximizing the user transmit rate and keeping the interference it causes to the FS system below a given limit. Following a similar approach as in [15], the optimal solution for this scenario can be obtained by solving the following multi-criteria optimization problem,

$$
\begin{array}{cl}
\max _{\mathbf{x}} & \mathbf{C} \\
\text { s.t. } & \mathbf{G p} \leq \mathbf{I} \\
& \mathbf{0} \leq \mathbf{p} \leq \mathbf{P}^{\max },
\end{array}
$$

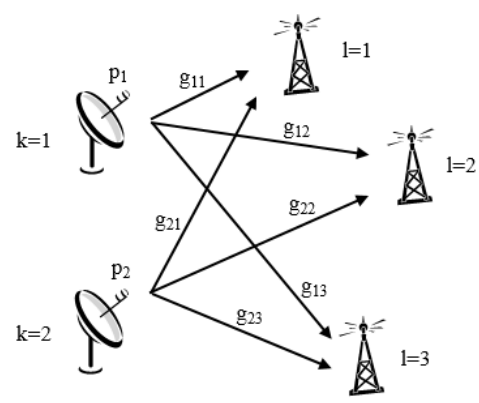

Fig. 2: Simplified scheme of cognitive satellite uplink network with $K=2$ FSS terminals and $L=3$ FS stations

where $\mathbf{C}=\left[\begin{array}{lll}C_{1} & \ldots & C_{K}\end{array}\right]^{\mathrm{T}}$ and the channel gains $g_{k, l}$ have been rearranged in matrix format as in (3).

$$
\mathbf{G}=\left[\begin{array}{ccc}
g_{1,1} & \cdots & g_{K, 1} \\
\vdots & \ddots & \vdots \\
g_{1, L} & \cdots & g_{K, L}
\end{array}\right]
$$

Vector $\mathbf{I}=\left[\begin{array}{lll}I_{1} & \ldots & I_{L}\end{array}\right]^{\mathrm{T}}$ in (2) includes the $L$ interference constraints required to guarantee the protection of the incumbent FS system. Such limitations are defined by the regulatory authorities. Typical reference limitations are given by ITU recommendations such as ITU-R F.758, where the interference level is recommended to be $-10 \mathrm{~dB}$ below the receiver noise.

The following sections devote to solve problem (2).

\section{Characterization of the Pareto Boundary}

Optimization problems involving more than one objective function to be optimized simultaneously, like (2), are known as Multi-objective Optimization Problems (MOP) [18]. A MOP problem usually admits infinite number of non-optimal solutions, which form a Pareto boundary and encompass a Pareto region [19]. The Pareto boundary establishes the outer boundary of the Pareto region, which are the working points where the performance cannot be improved for any user without exceeding interference constraints [20]. From (2) and (1), it is clear that each FSS terminal user aims at selfishly maximizing its own rate $C_{k}$ and altruistically consume the interference limit of the FS receivers. Throughput fairness can be introduce via game theory and pricing-based power control [21], but it is out of the scope of this paper to address this further. Therefore, it is optimal that each FSS terminal transmits with its maximum power obtained from the first constraint as long as the maximum allowable power is good enough to close the link, and not greater than the maximum power that the amplifier can support. In other words, the different objective functions do not conflict between themselves and thus the problem becomes,

$$
\begin{array}{cl}
\max _{\mathbf{p}} & \mathbf{p} \\
\text { s.t. } & \mathbf{G p} \leq \mathbf{I} \\
& \mathbf{0} \leq \mathbf{p} \leq \mathbf{P}^{\max } .
\end{array}
$$


Consequently, the Pareto rate points are obtained when the transmitters work at the edge of the Pareto power region. Fig. 3 illustrates the power and rate Pareto regions and the corresponding Pareto power and Pareto rate boundary for the satellite-terrestrial co-existence network depicted in Fig. 2 with,

$\mathbf{G}=\left[\begin{array}{cc}0.3 & 0.1 \\ 0.1 & 0.25 \\ 0.05 & 0.2\end{array}\right], \quad \mathbf{I}=\left[\begin{array}{l}2 \\ 2 \\ 2\end{array}\right], \quad \mathbf{P}^{\max }=\left[\begin{array}{l}10 \\ 10\end{array}\right], \quad d_{k}=1, \forall k$

From Fig. 3 one can observe that each FSS terminal is limited either by $P_{k}^{\max }$ or by the worst FS station in terms of received interference. This is evident by a glance of the maximum power $p_{k}$, when $p_{j}=0, \forall j \neq k$. For instance, this point is $p_{2}=8$ for user $k=2$, which is obtained identifying the worst FS receiver in terms of received interference (for user $k=2$ it is FS receiver $l=2$ with $g_{22}=0.25$ and $I_{2}=2$ ) and dividing the interference threshold of that receiver by the corresponding cross-channel power gain $\frac{I_{2}}{g_{22}}$. Also, the convexity of the Pareto region is determined by the crosschannel power gains $g_{k, l}$ contained in matrix G. Essentially, the Pareto region is convex when the most-upper right corner (indicated in Fig. 2 with $\mathbf{p}^{*}$ ) is above the line that connects the upper left corner and the lower right corner. If the Pareto region is convex, then the most-upper right corner provides the optimal solution to (2). High values of $g_{k, l}$ (interference links), however, might prevent the convexity of the Pareto region. In this case, interference management techniques such as beamforming or interference alignment [22] can be used to convexify the Pareto region.

In the next section we review and propose slight modifications of three different power allocation algorithms available in the literature in order to fulfill the received power mask with equality. That is the most upper right corner of the power region, $\mathbf{p}^{*}$ in Fig. 3(a).

\section{Power Allocation Techniques}

In this section, we first review the power allocation algorithm presented by the authors in [23]. In [23], a noniterative, simple and conservative technique that assumes perfect knowledge of cross-channel gains at the FSS system is proposed and it is shown to guarantee that the cognitive system never exceeds the prescribed interference threshold. Subsequently, we take the algorithm introduced in [14], [15] for TAS licensed and we performe slight adaptations for the scenario being considered in this paper. The latter, however, assumes the same side information as in [23]. Finally, a third technique is presented which works under the premise that the FS station is able to transmit 1-bit feedback indicating if the interference threshold has been exceeded or not. This 1-bit feedback from the incumbent system is sometimes refereed in the CR literature as "interference tweet" [24].

\section{A. Worst-Case Power Allocation}

The Worst-Case Power Allocation (WCPA) approach identifies the worst FS link per FSS terminal in terms of interference

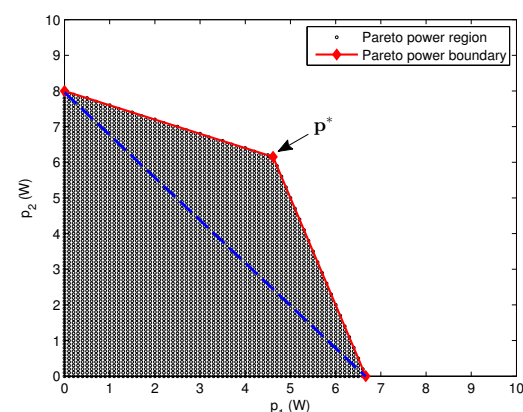

(a)

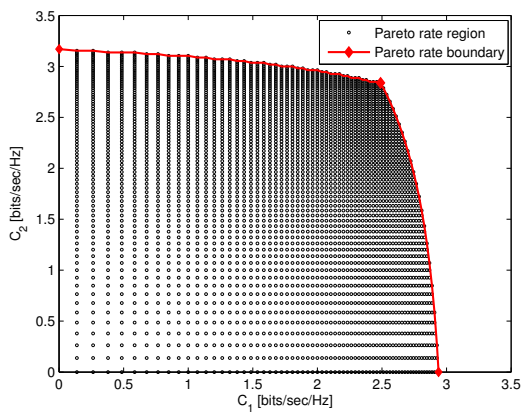

(b)

Fig. 3: Satellite-terrestrial co-existence network with $K=2$ FSS terminals and $L=3$ FS stations: (a) Power Pareto and feasible set, and (b) Rate Pareto and feasible set.

and divides the amount of tolerable interference among the total number of FSS terminals $K$. This requires complete acknowledge of cross channel link gains which can be obtained with a FS database and proper propagation modeling [23]. The identification of the worst FS station in terms of interference consists in determining the one with maximum cross-channel gain,

$$
l_{k}=\max _{l}[\mathbf{G}]_{k},
$$

Next, the interference limit of the worst FS receiver, $I_{l_{k}}$, is used to establish the maximum transmit power as follows,

$$
p_{k}=\frac{I_{l_{k}}}{K \cdot g_{k, l_{k}}} \text {. }
$$

\section{B. Gradient-Based Power Control (GBPC)}

Under the premise that cross channel gains are known at the FSS system, we consider the following iterative power updating rule for each $k$-th FSS terminal,

$$
p_{k}(n)=p_{k}(n-1)+\frac{\alpha}{g_{k, l_{k}}}\left(I_{l_{k}}-P_{l_{k}}^{R x}(n-1)\right),
$$

where $l_{k}$ is obtained from (6) and $P_{l_{k}}^{R x}(i)$ is the total amount of received power at the $l_{k}$ FS station at the $i$-th time instant, which can be obtained selecting the $l_{k}$-th component of the vector resulting from the product $\mathbf{G p}$. $\alpha$ is a control gain whose purpose is to control the fluctuations and convergence of the method. The power updating rule proposed in (8) slightly 
differs from the one presented in [14], [15] in the fact that the rates considered in the multi-objective problem (2) do not have an interference component caused by the transmitted powers $p_{k}$ to be optimized. Therefore, the direct links $d_{k}$, $k=1, \ldots, K$, are not taken into account in the computation of $P_{l_{k}}^{R x}$ neither in the denominator of the updating rule. Moreover, [14], [15] consider restrictions in the total received power while here we use an interference constraint limit [12].

Note that this power allocation has to be performed at the Network Control Center (NCC) of the FSS system since all transmit powers $x_{k}$ need to be known to compute $P_{l_{k}}^{R x}(i)$. Clearly, both (7) and (8) are very difficult to implement in practice, particularly for the channel state information from the cognitive transmitter to the incumbent receiver.

\section{1-bit Feedback GBPC}

This technique assumes that the FS receivers send 1-bit feedback: "0" and "1" indicating that the received interference is "below" or "above" the interference threshold. We propose an heuristic approach for solving the power allocation with a minimal number of interference violations. A similar algorithm is proposed for a $2 \times 2$ terrestrial cognitive system in [25], where the cognitive user constantly monitors the modulation and coding scheme (MODCOD) used by the incumbent user. Here, we modify [25] based on the feedback received from the incumbent FS system. Algorithm 1 summarizes the proposed 1-bit Feedback Gradient-Based Power Control approach.

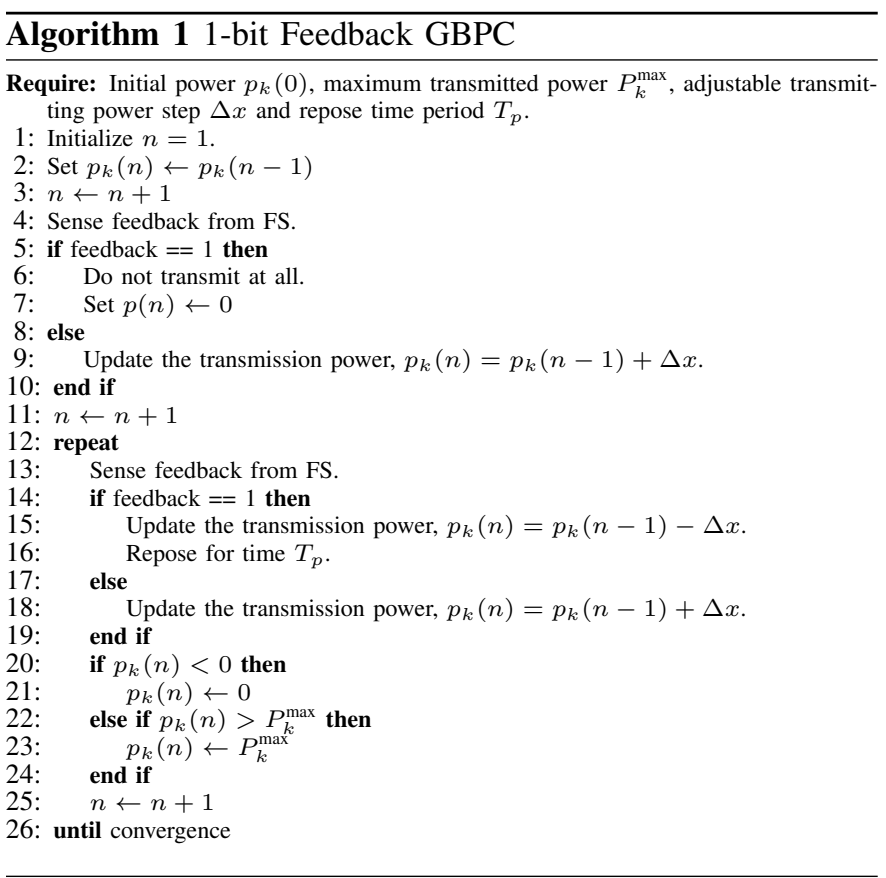

Essentially, the FSS terminal starts transmitting the initial transmit power and then gradually boosts it until either it achieves $P_{k}^{\max }$ or a violation occurs. The increasing step is determined by $\Delta x$, which should be small enough in order to achieve convergence and high enough to speed up the convergence. After every interference violation, the FSS terminal reduces the power to the precedent value and reposes for a period $T_{p}$, and after that it tries to increase it again. Regarding $T_{p}$, the spectrum utilization by FS links is almost constant in time and the FSS terminals are assumed fixed, then we can assume very few variations will occur in a certain amount of time. This means that, once the first violation occurs and the emitted power is adjusted, we do not have to keep checking the feedback so often (i.e., $T_{p}$ high). Eventually, each FSS terminal converges to a value below $P_{k}^{\max }$ without breaching the interference limit many times.

\section{Simulation Results}

In order to evaluate the three power control methods, Fig. 4(a) and Fig. 4(b) depict the evolution of power and rate, respectively, for the GBPC and 1-bit feedback GBPC presented in the previous section for the same network considered in Fig. 3 with $\alpha=0.05, T_{p}=20$ iterations and $\Delta x=0.5$. The result of the WCPA is also depicted on the figures for the sake of comparison. The FSS terminal emitted power evolution and the received power evolution at each FS receiver are shown in Fig. 5(a) and Fig. 5(b), respectively, for the same two-user network.

Clearly, the worst-case technique proposed in [23] is too conservative in the sense that it achieves a working point that satisfies the interference constraints but it is far away from the optimal point, $\mathbf{p}^{*}$. The two gradient-based techniques perform much better achieving in terms of optimality. The GBPC achieves the optimal working point without ever exceeding the interference thresholds. The intermediate approach that assumes 1-bit feedback converges to a working point close to the optimal one but exceeding the interference threshold few times. Therefore, as expected, the results confirm the fact that the availability of channel state information helps in speeding up convergence while never exceeding the prescribed interference limits.

\section{CONCLUSION}

This paper has presented three different efficient power allocation algorithms for the cognitive uplink satellite-terrestrial co-existence. The conclusion extracted from the numerical results is that there is a clear tradeoff between the level of channel state information needed at the satellite system and the rates that can be achieved at the cognitive satellite network.

\section{ACKNOWLEDGMENT}

This work was partially supported by the European Commission in the framework of the FP7 CoRaSat (Grant agreement no. 316779) and the H2020 SANSA project (Grant agreement no. 645047) and by the National Research Fund, Luxembourg, under CORE project SeMIGod, and CORE project SATSENT.

\section{REFERENCES}

[1] EU, "Digital agenda for europe," http://ec.europa.eu/digital-agenda.

[2] O. Johnson, G. Peters, and G. Jones, "The EU Digital Agenda: Modelling the opportunity for satellite delivery," IEEE AESS European Conf. on Satellite Telecommunications (ESTEL), Rome, Italy, Oct, 2012. 


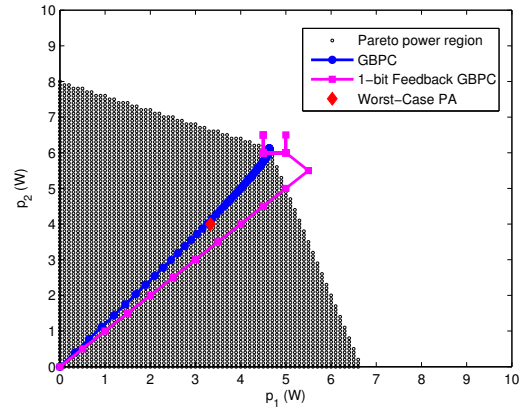

(a)

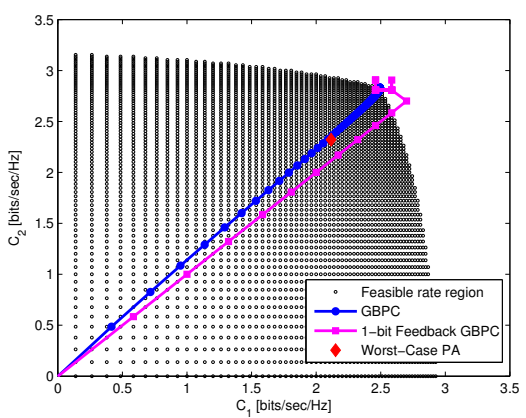

(b)

Fig. 4: Convergence of the iterative techniques and the result of WCPA for the example in Fig. 3. (a) Power feasible set and convergence of the techniques, and (b) Rate feasible set and convergence of the techniques.

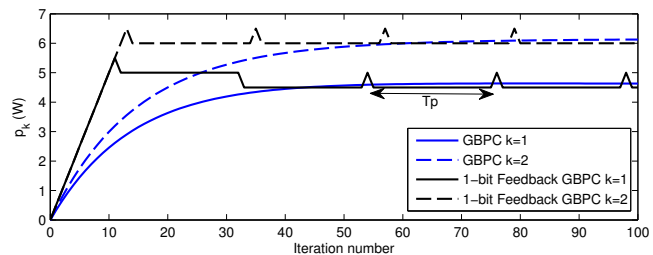

(a)

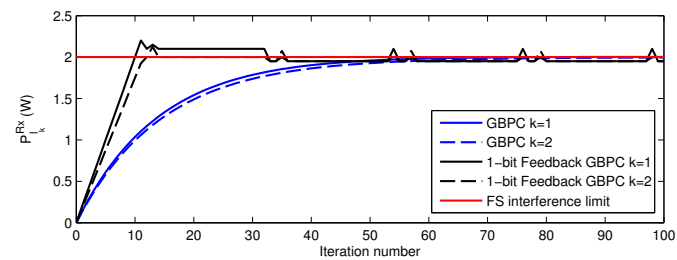

(b)

Fig. 5: Convergence of the two gradient-based techniques for the same scenario considered in Fig. 4. (a) Emitted power evolution, and (b) Received power evolution.

[3] A. Kyrgiazos, B. Evans, P. Thompson, P.T. Mathiopoulos, and S. Papaharalabos, "A Terabit/Second Satellite System for European Broadband Access: A Feasibility Study," Int. J. Satell. Commun. Network., vol. 32, no. 2, pp. 63-92, Mar, 2014.

[4] E. Biglieri, A.J. Goldsmith, L.J. Greenstein, N.B. Mandayam, and H.V. Poor, Principles of Cognitive Radio, Cambridge University Press, Cambridge, UK, Dec, 2012.
[5] H2020 project, "Shared Access terrestrial-satellite backhaul Network enabled by Smart Antennas - SANSA," http://sansa-h2020.eu.

[6] FP7 project, "COgnitive RAdio for SATellite Communications CoRaSat," http://www.ict-corasat.eu.

[7] S. Maleki, S. Chatzinotas, B.G. Evans, K. Liolis, J. Grotz, A. VanelliCoralli, and N. Chuberre, "Cognitive Spectrum Utilization in Ka Band Multibeam Satellite Communications," IEEE Communication Magazine, vol. 53, no. 3, pp. 24-29, Mar, 2015.

[8] M. Hoyhtya, J. Kyrolainen, A. Hulkkonen, J. Ylitalo, and A. Roivainen, "Application of Cognitive Radio Techniques to Satellite Communication," IEEE Int. Symp. on Dynamic Spectrum Access Networks (DYSPAN), Bellevue, USA, Oct, 2012.

[9] M.A. Vázquez, A. Pérez-Neira, and M.A. Lagunas, "Linear Precoding in Multibeam Satellite under Licensed Shared Access," Adv. Satellite Multimedia Systems Conf. (ASMS) and Signal Process. for Space Commun. Workshop (SPSC), Livorno, Italy, Sep, 2014.

[10] K. Tourki, K.A. Qaraqe, and M.S. Alouini, "Outage Analysis for Underlay Cognitive Networks Using Incremental Regenerative Relaying," IEEE Trans. Veh. Technol., vol. 62, no. 2, pp. 721-734, Feb, 2013.

[11] R. Zhang, "On Peak versus Average Interference Power Constraints for Protecting Primary Users in Cognitive Radio Networks," IEEE Trans. Wireless Commun., vol. 8, no. 4, pp. 2112-2120, Apr, 2009.

[12] Y.Y. He and S. Dey, "Throughput Maximization in Cognitive Radio Under Peak Interference Constraints With Limited Feedback," IEEE Trans. Veh. Technol., vol. 61, no. 3, pp. 1287-1305, Mar, 2012.

[13] S. Vassaki, M. Poulakis, A.D. Panagopoulos, and P. Constantinou, "Optimal Power Allocation under QoS Constraints in Cognitive Radio Systems," Int. Symp. on Wireless Communication Systems (ISWCS), Aachen, Germany, pp. 552-556, Nov,2011.

[14] A. Pérez-Neira, J.M. Veciana, M.A. Vázquez, and E. Lagunas, "Distributed Power Control with Received Power Constraints for Time-AreaSpectrum Licenses," Signal Processing, under review.

[15] A. Pérez-Neira, M.A. Lagunas, and M.A. Vázquez, "Autonomous Design for the MISO Interference Channel with Mask Constraints at Reception," IEEE Applied Electromagnetics Conf. (AEMC), Kolkata, India, Dec, 2011.

[16] S.K. Sharma, S. Chatzinotas, and B. Ottersten, "Satellite Cognitive Communications: Interference Modeling and Techniques Selection," Adv. Satellite Multimedia Systems Conf. (ASMS) and Signal Process. for Space Commun. Workshop (SPSC), Baiona, Spain, Sep, 2012.

[17] A. Mohamed, M. Lopez-Benitez, and B. Evans, "Ka Band Satellite Terrestrial Co-Existence: A Statistical Modelling Approach," Ka and Broadband Communications, Navigation and Earth Observation Conf., Salerno, Italy, Oct, 2014.

[18] E. Bjornson, E. Jorswieck, M. Debbah, and B. Ottersten, "Multiobjective Signal Processing Optimization: The Way to Balance Conflicting Metrics in 5G Systems," IEEE Signal Process. Mag., vol. 31, no. 6, pp. 14-23, Nov, 2014.

[19] L. Zadeh, "Optimality and Non-Scalar-Valued Performance Criteria," IEEE Trans. Automat. Contr., vol. 8, no. 1, pp. 59-60, Jan, 1963.

[20] E. Bjornson, M. Bengtsson, and B. Ottersten, "Pareto Characterization of the Multicell MIMO Performance Region With Simple Receivers," IEEE Trans. Signal Process., vol. 60, no. 8, pp. 4464-4469, Aug, 2012.

[21] C.G. Yang, J.D. Li, and Z. Tian, "Optimal Power Control for Cognitive Radio Networks Under Coupled Interference Constraints: A Cooperative Game-Theoretic Perspective," IEEE Trans. Veh. Technol., vol. 39, no. 4, pp. 1696-1706, May, 2010.

[22] G. Bresler, D. Cartwright, and D. Tse, "Feasibility of Interference Alignment for the MIMO Interference Channel," IEEE Trans. Inf. Theory, vol. 60, no. 9, pp. 5573-5586, Sep, 2014.

[23] E. Lagunas, S.K. Sharma, S. Maleki, S. Chatzinotas, J. Grotz, J. Krause, and B. Ottersten, "Resource Allocation for Cognitive Satellite Uplink and Fixed-Service Terrestrial Coexistence in Ka-band," Int. Conf. on Cognitive Radio Oriented Wireless Networks (CROWNCOM), Doha, Qatar, Apr, 2015.

[24] A. Marques, E. Dall'Anese, and G.B. Giannakis, "Cross-Layer Optimization and Receiver Localization for Cognitive Networks Using Interference Tweets," IEEE J. Sel. Areas Commun., vol. 32, no. 3, pp. 641-653, Mar, 2014.

[25] A. Tsakmalis, S. Chatzinotas, and B. Ottersten, "Modulation and Coding Classification for Adaptive Power Control in 5G Cognitive Communications," IEEE Int. Workshop on Signal Process. Adv. in Wireless Comm. (SPAWC), Toronto, Canada, Jun, 2014. 